\title{
Intraarterial and intravenous contrast enhanced CT and MR imaging of multi-step hepatocarcinogenesis defining the early stage of hepatocellular carcinoma development
}

\author{
Satoshi Kobayashi' ${ }^{1,2}$, Kazuto Kozaka ${ }^{2}$, Toshifumi Gabata ${ }^{2}$, Osamu Matsui², Tetsuya Minami ${ }^{3}$ \\ 'Department of Quantum Medical Technology, Kanazawa University Graduate School of Medical Sciences, Kanazawa 920- \\ 0942, Japan. \\ 2Department of Radiology, Kanazawa University Graduate School of Medical Sciences, Kanazawa 920-8641, Japan. \\ ${ }^{3}$ Department of Radiology, Kanazawa Medical University, Uchinada 920-0293, Japan.
}

\begin{abstract}
Correspondence to: Dr. Satoshi Kobayashi, Department of Radiology, Kanazawa University Graduate School of Medical Sciences, 13-1, Takara-machi, Kanazawa920-8641, Japan. E-mail: satoshik@staff.kanazawa-u.ac.jp
\end{abstract}

How to cite this article: Kobayashi S, Kozaka K, Gabata T, Matsui O, Minami T. Intraarterial and intravenous contrast enhanced CT and MR imaging of multi-step hepatocarcinogenesis defining the early stage of hepatocellular carcinoma development. Hepatoma Res 2020;6:36. http://dx.doi.org/10.20517/2394-5079.2020.24

Received: 11 Mar 2020 First Decision: 7 Apr 2020 Revised: 22 Apr 2020 Accepted: 26 Apr 2020 Published: 18 Jun 2020

Science Editor: Yuko Kono Copy Editor: Jing-Wen Zhang Production Editor: Tian Zhang

\begin{abstract}
Liver cancer is the second leading cause of cancer deaths in men worldwide, and the 6th and 7th cause of cancer deaths in men and women in developed countries. 70\%-90\% of primary liver cancer is hepatocellular carcinoma. Hepatitis B or C viruses and chronic inflammation due to alcohol intake are the main risk factors for hepatocellular carcinoma. One of the key approaches for the early detection of hepatocellular carcinoma is to understand the specific imaging findings of liver nodules in the multi-step hepatocrcinogenesis process. In this article, we review the imaging findings of multistep hepatocarcinogenesis, with a focus on the early detection of malignant, cirrhotic nodules with $\mathrm{CT}$ and $\mathrm{MRI}$.
\end{abstract}

Keywords: Hepatocellular carcinoma, multistep hepatocarcinogenesis, early hepatocellular carcinoma, dysplastic nodule, CT, MRI

\section{INTRODUCTION}

Liver cancer is the second and 6th leading cause of cancer deaths in men and women worldwide, and the 6th and 7th cause of cancer deaths in men and women in developed countries. In 2012, the annual incidence and mortality rates were approximately 780,000 and 750,000 respectively ${ }^{[1]}$.

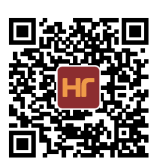


70\%-90\% of primary liver cancer is hepatocellular carcinoma (HCC). Hepatitis B or C virus infections and chronic inflammation due to alcohol intake are the main risk factors of HCC. In Japan, such high-risk patients have been identified and early detection and treatment systems have been established.

One of the key approaches for the early detection of HCC is to understand its characteristic imaging findings of nodules undergoing the multi-step hepatocrcinogenesis process. In other words, picking up early HCC within various types of pre-malignant nodules in cirrhotic livers is essential for the early detection of clinically significant HCC.

For such early detection, contrast enhanced CT, MRI and contrast enhanced ultrasound (CE-US) might be used as imaging modalities. In particular, recent advancements in ultrasonographic contrast agents have enabled monitoring of detailed haemodynamics and Kupffer cell function of liver nodules. Compared to CT and MRI, CE-US is a powerful tool for the analysis of multistep hepatocarcinigenesis because it enables real time monitoring of the dynamics of the contrast agent within the liver. However, we do not have enough experience to discuss the advantages of CE-US in multi-step hepatocarcinogenesis. Therefore, in this article, we will review the imaging findings of multi-step hepatocarcinogenesis on CT and MRI instead, and to focus on the early detection of malignant cirrhotic nodules.

\section{MULTI-STEP HEPATOCARCINOGENESIS}

Hepatocarcinogenesis exhibits a multi-step process. It starts from a regenerative nodule in cirrhosis or as a dysplastic nodule $(\mathrm{DN})$, which is a precancerous condition, and progresses to advanced HCC [Figure 1$]^{[2]}$. A considerable proportion of HCC arise through multi-step hepatocarcinogenesis while other HCCs develop de-novo ${ }^{[2,3]}$.

Therefore, in high-risk patients, it is important to monitor the process of malignant transformation from precancerous nodules to overt malignant nodule in HCC. Treatment of malignant nodules in early stages might thus be expected to lead to an improved prognosis for HCC patients if detected early.

\section{PATHOLOGICAL DIFFERENTIATION OF MALIGNANT FROM BENIGN NODULES}

Histopathologically, the multi-step hepatocarcinogenesis process is observed when moderately or poorly differentiated HCC (advanced HCC) develops in the nodule of well differentiated HCC [Figure 1] $]^{[4]}$. This nodule-in-nodule appearance differs however, when considering definitions in pathology (malignant focus in premalignant nodule) and imaging (hypervascular focus in nonhypervascular nodule). Similar nodule-innodule structures are also observed in diagnostic imaging although the detection rate is not high in routine CT and MRI. However, when the characteristics of the inner nodule are different from the surrounding nodule, such as a hypervascular foci within a hypovascular nodule (as discussed later), or the absence of a superparamagnetic iron oxide (SPIO) uptake foci within the SPIO-uptake nodule, these different findings within the same nodule would represent imaging findings of multi-step hepatocellular carcinogenesis [Figure 2].

Pathologically, DNs are classified into low grade DN (LGDN), which is close to normal, and high grade DN (HGDN), which is close to malignancy. The nodule that is histologically more malignant than HGDN is called early HCC. The clinical and pathological characteristics of the nodules which develop in multi-step hepatocarcinogenesis in cirrhosis are shown in Figure $3^{[5,6]}$.

To differentiate HGDN from early HCC histologically, the findings of tumor cell invasion in the remaining portal tract (stromal invasion) is an important hallmark of early $\mathrm{HCC}^{[5,7]}$. 


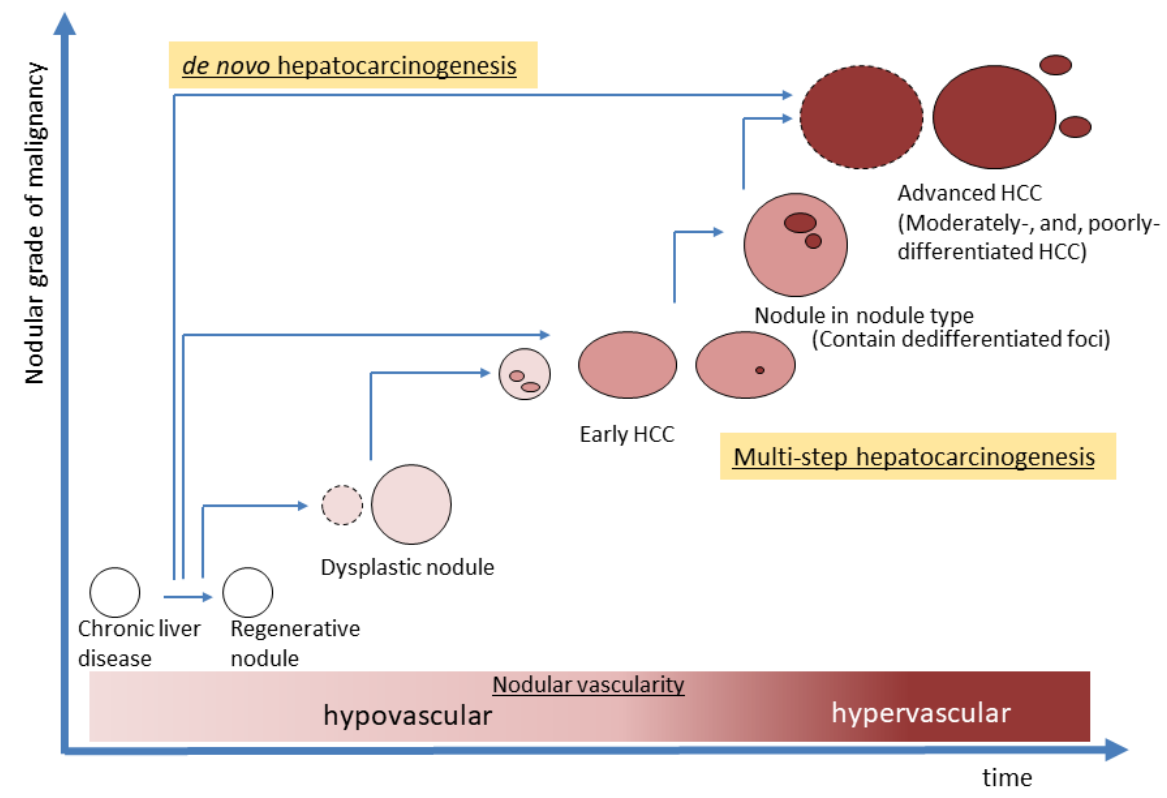

Figure 1. Schematic diagram of multistep hepatocarcinogenesis. Two types of human hepatocarcinogenesis are currently considered, one is multistep- and the other is de novo-hepatocarcinogenesis. In multistep hepatocarcinogenesis, the nodular grade of malignancy changes from dysplastic nodule to advanced HCC stepwisely, and nodular vascularity also gradually change from hypovascular to hypervascular. HCC: hepatocellular carcinoma
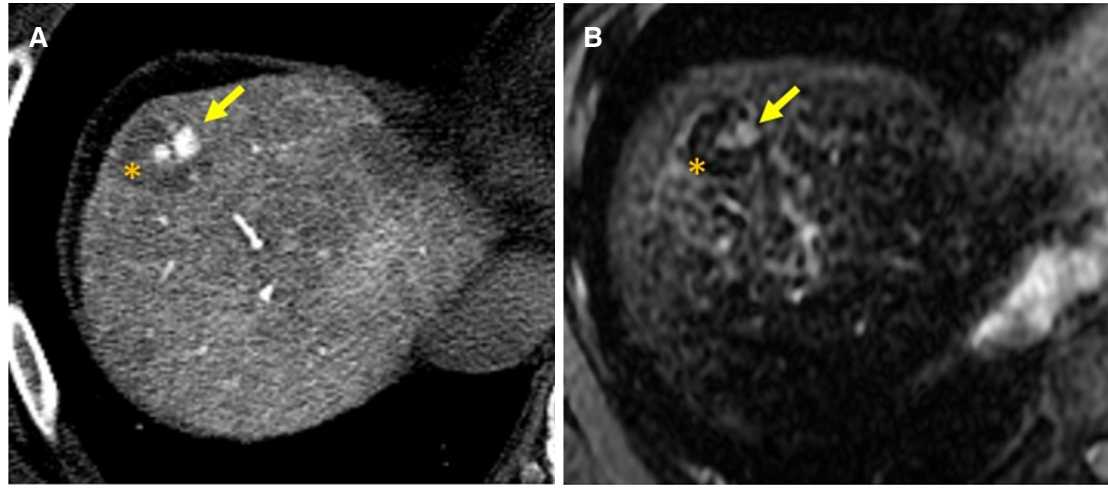

Figure 2. Example of nodule in nodule appearance lesion in alcoholic cirrhosis. A: CT during hepatic arteriography image of nodule in nodule lesion. Some hypervascular foci (arrow) are observed within hypovascular nodule $\left({ }^{*}\right.$ ); B: SPIO enhanced T2-MR image of nodule in nodule lesion. Small hyperintense spots, which do not uptake SPIO (arrow), ate observed within the nodule which show hypointensity because of uptake of SPIO $\left(^{*}\right)$. These findings represent the visualization of multistep hepatocarcinogenesis within the nodule. SPIO: superparamagnetic iron oxide

Macroscopically, HGDN and early HCC both show ill-defined nodules and are difficult to differentiate. Histologically, both show increased cell density but are scarce in structural atypia or cellular atypia. Therefore, stromal invasion of atypical cells is important for differentiating HGDN and early HCC on histopathology ${ }^{[5]}$.

When a histological study is performed in the portal tract of a completely excised nodule, differentiation of HGDN or early HCC is possible [Figure 4]. In a biopsy specimen however, misdiagnosis of the early HCC nodule as HGDN can happen because the specimen is small and does not contain sufficient portal tracts [Figure 4]. In diagnosis based on imaging, findings reflect the total image of the nodule and the presence of stromal invasion of atypical cells might not be shown.

For these reasons, it is inevitable that borderline cases on histology between HCC and DN is different from that seen on imaging. 


\begin{tabular}{|c|c|c|c|c|}
\hline $\begin{array}{l}\text { Portal tract } \\
\text { (contain hepatic } \\
\text { arterial and portal } \\
\text { branch) }\end{array}$ & $\begin{array}{l}\text { Low-grade } \\
\text { dysplastic } \\
\text { nodule }\end{array}$ & $\begin{array}{l}\text { High-grade } \\
\text { dysplastic } \\
\text { nodule }\end{array}$ & $\begin{array}{l}\text { Well differentiated } \\
\mathrm{HCC}\end{array}$ & $\begin{array}{l}\text { Moderately } \\
\text { Differentiated HCC }\end{array}$ \\
\hline \multicolumn{5}{|l|}{ Pathological features } \\
\hline gross appearance & & & vaguely-nodular & $\begin{array}{l}\text { distinctly- } \\
\text { nodular }\end{array}$ \\
\hline stromal invasion & $(-)$ & $(-)$ & $+/-$ & $+/-$ \\
\hline \multicolumn{5}{|l|}{ Imaging findings } \\
\hline arterial supply & iso/ hypo & iso/ hypo & $\begin{array}{l}\text { iso/ hypo } \\
\text { rarely hyper }\end{array}$ & hyper \\
\hline portal venous supply & + & + & + & - \\
\hline Clinico-pathological & \multicolumn{2}{|c|}{ Premalignant } & Early HCC & Overt HCC \\
\hline
\end{tabular}

Figure 3. Clinical and pathological characteristics of small nodular lesions during multistep hepatocarcinogenesis step in cirrhotic liver. HCC: hepatocellular carcinoma
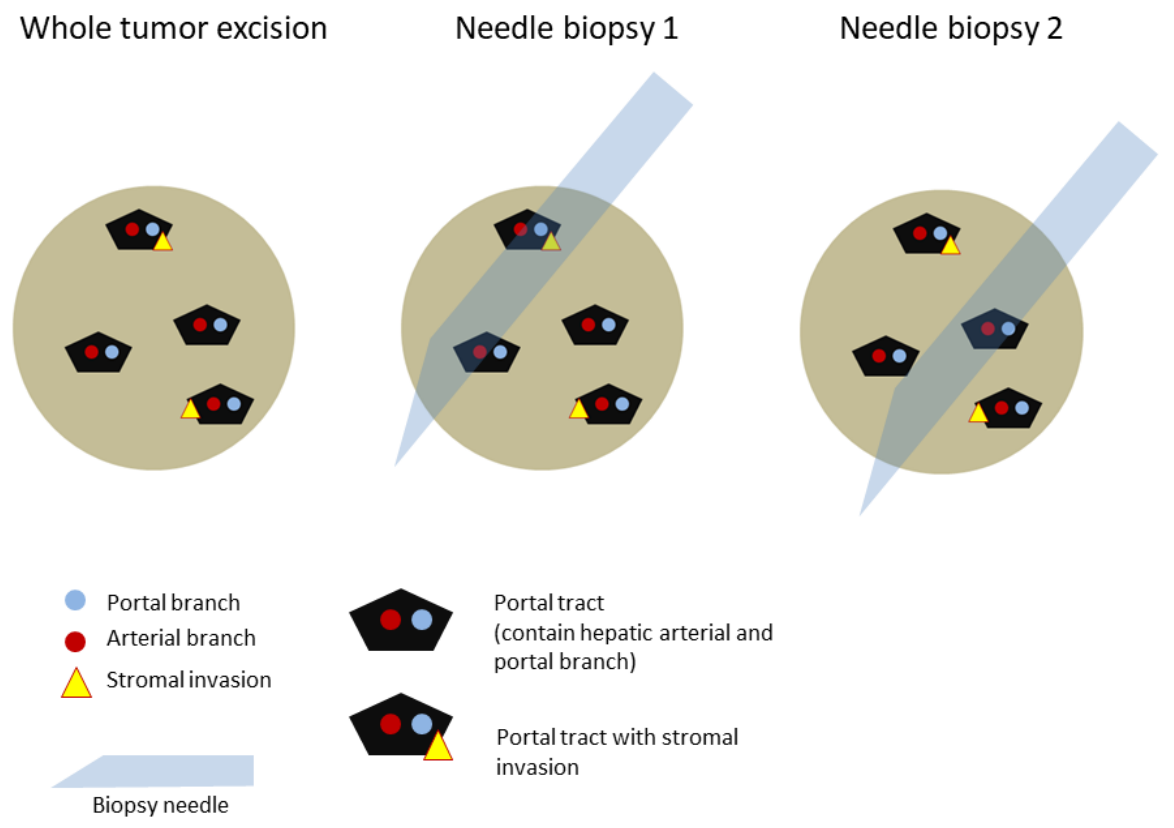

Figure 4. Problem in histological diagnosis of early HCC with needle biopsy specimen. Histopathologically, the presence of the stromal invasion of tumor cell to the portal tract is the most reliable marker for the diagnosis of early HCC. In whole tumor excision specimen, and needle biopsy 1 specimen, pathologist can find out the stromal invasion within the specimen and diagnose the nodule as early HCC. However, in needle biopsy 2, the stromal invasion finding is not contained within the specimen and pathologist diagnose the nodule as high-grade dysplastic nodule, not early HCC. This kind of sampling error issue is possible in histopathological diagnosis of the nodule during multistep hepatocarcinigenesis. HCC: hepatocellular carcinoma

It is impossible however, to excise all nodules in liver cirrhosis to identify HGDN or early HCC. In addition, biopsies could be misdiagnosed due to sampling error. Therefore, it is reasonable to attempt differentiation between benign and malignant nodules through imaging with CT and MRI, which are relatively minimally invasive compared to histopathology. 

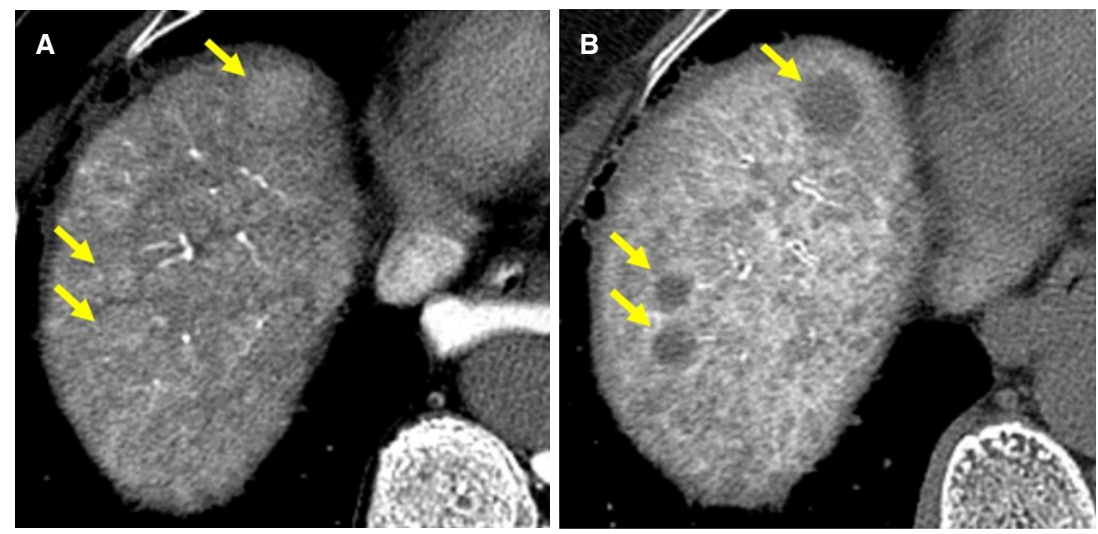

Figure 5. Example of high grade dysplastic nodule in cirrhotic liver. A: on CT during arterial portography, there are isodense nodule compared to background liver (arrows), which represent nodular portal flow is preserved; B: on CT during hepatic arteriography, these nodules show hypodensity compared to the background liver (arrows), which indicate the nodular arterial supply is decreased. This hypovascular pattern represents relatively benign nature nodules under multistep hepatocarcinogenesis

The different imaging modalities however, reflect different pathophysiological features between precancerous conditions (benign) and cancerous nodules (malignant), so the same nodule may be assessed differently through different modalities. Therefore, sound judgment is necessary based on the findings of multiple modalities when there is discrepancy.

\section{VISUALIZATION OF MULTI-STEP HEPATOCARCINOGENESIS ON IMAGING AND DIFFERENTIATION OF BENIGN AND MALIGNANT NODULES}

\section{Approach by haemodynamic imaging}

Histological studies have found that during multi-step hepatocarcinogenesis, the number of portal tracts in nodules gradually decreases as the nodular grade of malignancy changes from DN to hypervascular, classical HCC, and the number of arteries which do not run with portal veins (unpaired artery) increases [Figure 3] ${ }^{[8]}$.

Angiography-assisted CT, which selectively infuses a contrast agent into the hepatic artery or portal vein during CT scan of the liver, can evaluate the degree of arterial and portal blood supply to the nodule. Angiography-assisted CT consists of the following two methods: one is CT during hepatic arteriography (CTHA), which evaluates hepatic arterial blood supply, and the other is CT during arterial portography (CTAP), which evaluates portal venous blood supply. We can evaluate the degree of hepatic arterial flow and portal venous flow of the nodules undergoing multi-step hepatocarcinogenesis on CTHA and CTAP. With angiography-assisted CT, we have found that LGDN, HGDN, and early HCC, which are all relatively benign nodules, were hypovascular in nature with low arterial and preserved portal blood flow [Figure 5]. In contrast, more malignant nodules, such as well differentiated HCC and moderately differentiated HCC, showed a hypervascular nature with low portal blood flow and high arterial blood flow [Figure 6] ${ }^{[9]}$.

These imaging findings reflect the histological changes of the nodules during multistep hepatocarcinogenesis: the number of portal tracts, which contain hepatic arteries and portal veins, decreases as the nodular grade of malignancy increases, and at some point, unpaired arteries starts to develop within the nodule. Figure 7 shows the relationship between the nodular grade of malignancy and the degree of hepatic arterial and portal blood supply of nodules.

Recently, Kitao et al. ${ }^{[10]}$ showed that drainage vessels of the nodules during multistep hepatocarcinogenesis process transform as the nodular grade of malignancy changes [Figure 8]. They compared angiographyasisted CT findings with histological findings of the nodules during multistep hepatocarcinogenesis and 

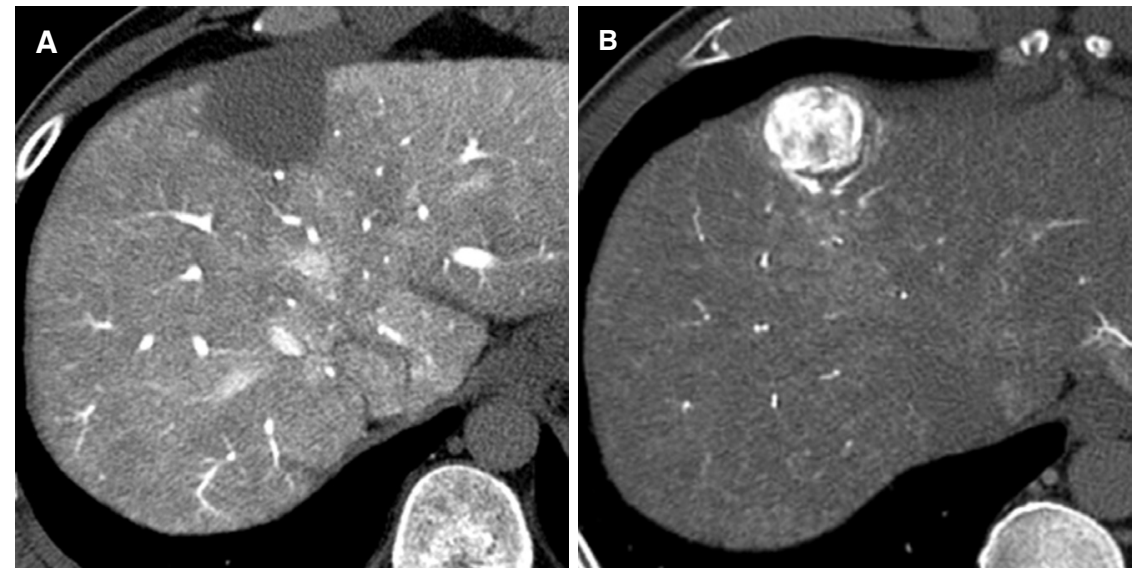

Figure 6. Example of advanced (moderately differentiated) hepatocellular carcinoma in cirrhotic liver. A: on CT during arterial portography, there are hypodense nodule compared to background liver, which represent loss of nodular portal flow; B: on CT during hepatic arteriography, the lesion shows marked hyperdensity compared to the background liver, which indicate increased nodular arterial flow. This hypervascular pattern indicates definite malignant nodule such as moderately differentiated hepatocellular carcinoma

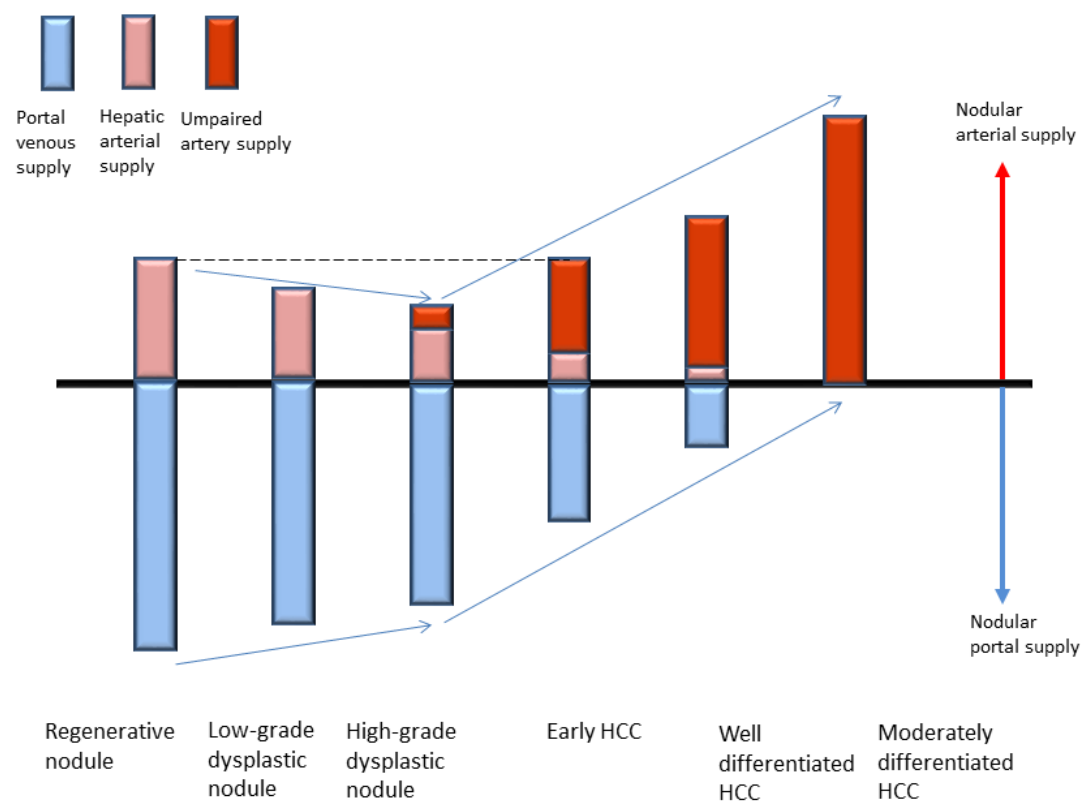

Figure 7. Relationship between the nodular grade of malignancy and the degree of intranodular blood supply of the nodule. HCC: hepatocellular carcinoma

found that in hypovascular nodules, such as DN and early HCC in which portal blood flow is maintained and the increase in arterial blood supply not seen yet, blood that drains from the tumor flows from tumor sinusoids into intranodular hepatic venules and then into extranodular hepatic veins. In contrast, in hypervascular nodules, especially in HCC without capsules, blood flows from within tumor sinusoids into the surrounding hepatic sinusoids; and in hypervascular HCC with capsules, blood flows from within tumor sinusoids into intracapsule portal venules, and then into surrounding hepatic sinusoids.

These findings show that during hepatocarcinogenesis, the hepatic vein is initially occluded by increased intratumoral pressure caused by increase in cell density, and direct connections between tumor sinusoids and surrounding hepatic sinusoids work as the tumor drainage route because of occlusion of the hepatic vein. Then, as the nodular grade of malignancy progresses, the tumor becomes encapsulated and the direct 

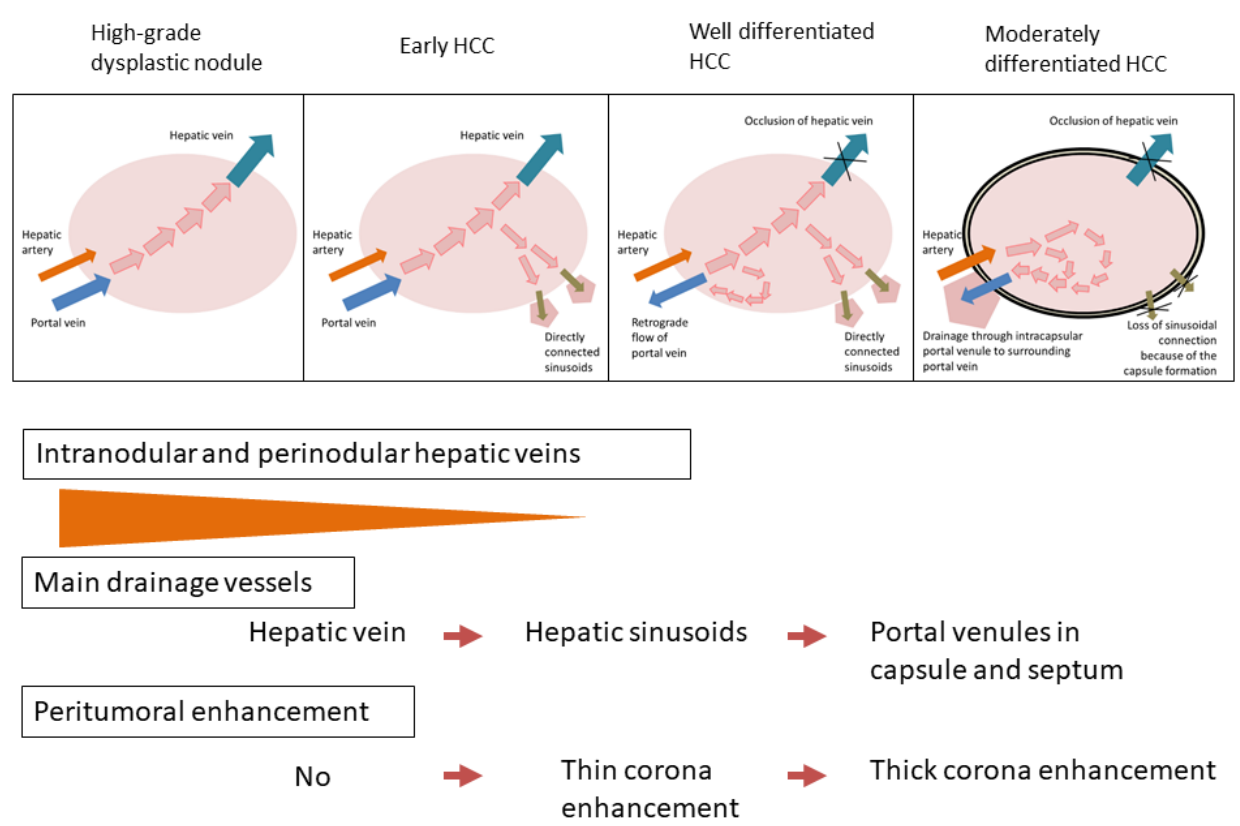

Figure 8. Multistep changes of drainage vessels and peritumoral enhancement during hepatocarcinogenesis. HCC: hepatocellular carcinoma
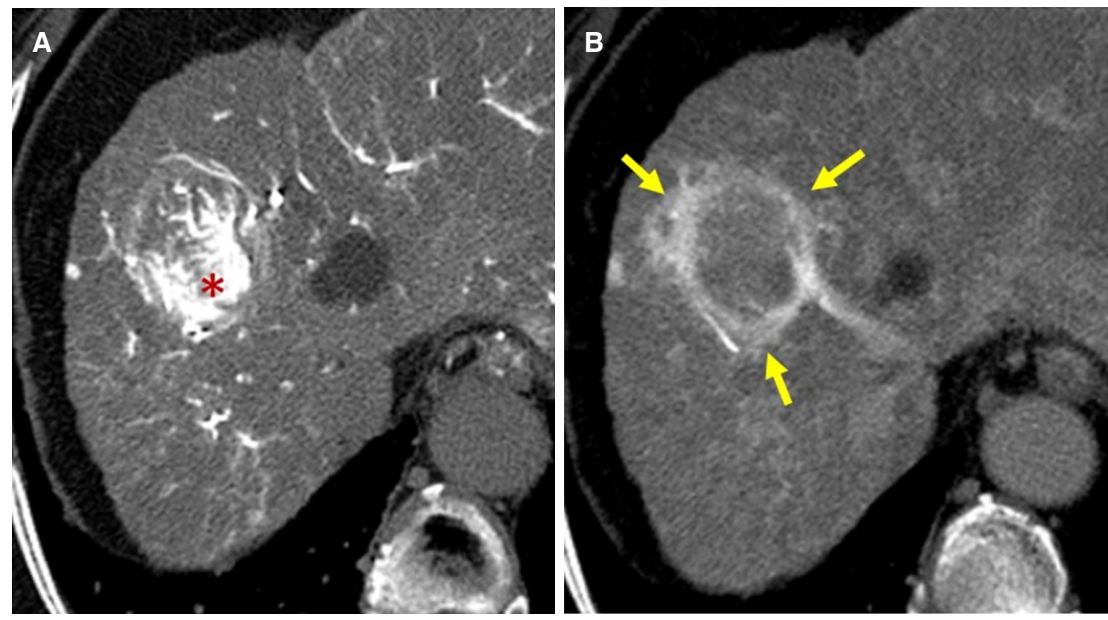

Figure 9. Example of corona enhancement caused by drainage flow from hypervascular HCC. A: on early phase image of CT during hepatic arteriography, HCC shows marked enhancement because of hypervascularity $\left(^{*}\right)$; B: on late phase image of $\mathrm{CT}$ during hepatic arteriography, since the intraarterial contrast injection is stopped, the enhancement of HCC decreases $\left(^{\star}\right)$ and peritumoral mormal liver parenchyma is enhanced by drainage of contrast material from HCC (arrow). HCC: hepatocellular carcinoma

connection between tumor sinusoids and surrounding hepatic sinusoids is lost, such that drainage flow is retrograde through the intracapsular portal venules. On CTHA, this retrograde flow of contrast medium stains surrounding hepatic parenchyma around the tumor and is identified as corona enhancement [Figure 9] ${ }^{[11]}$.

On angiography-assisted CT, LGDN, early HCC, and part of well-differentiated HCC are all observed as hypovascular nodules. In contrast, the presence of a nodule-in-nodule appearance, which has a partly hypervascular foci within a hypovascular nodule, is a definite hallmark to discriminate between hypovascular, relatively benign nodules and more malignant nodules since the latter display hypervascularity [Figure 10]. In hypovascular nodules, most show iso-density on CTAP and hypo-density on CTHA since nodular portal blood flow is preserved and increase in hepatic arterial flow has not started, which represents 

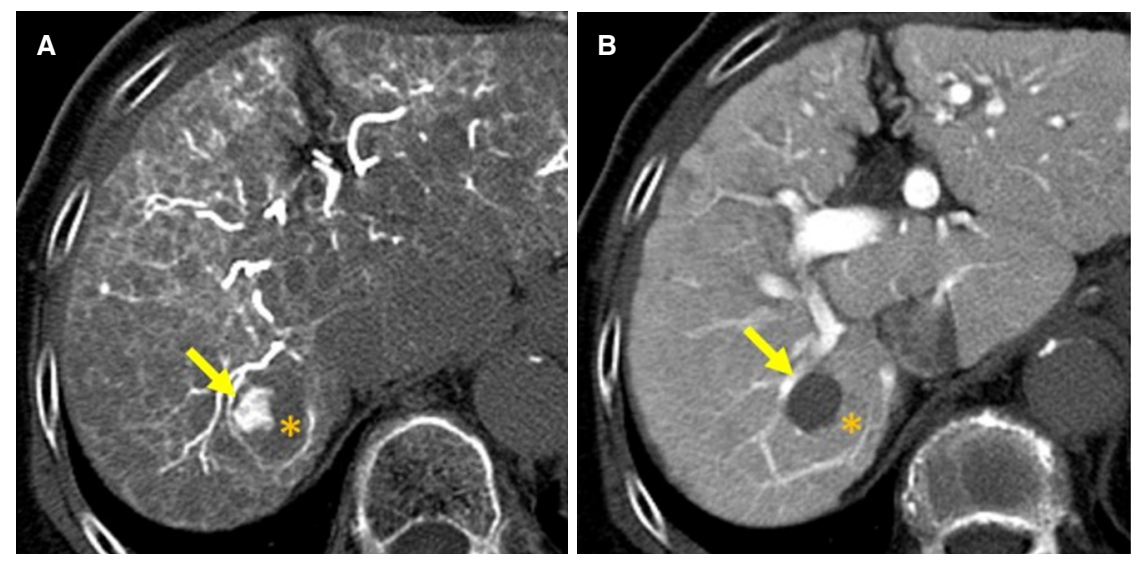

Figure 10. Hypovascular nodule with hypervascular foci (nodule in nodule appearance lesion). A: on CT during hepatic arteriography, hypervascular foci (arrow) is observed within hypovascular nodule (*); B: on CT during arterial portography, only hypervascular portion on CT during hepatic arteriography (arrow) shows hypointensity and other portion of the nodule show isointensity to the background liver $\left(^{\star}\right)$. In this nodule hypovascular portion of the nodule $\left(^{\star}\right)$ are relatively benign nature and hypervascular foci represents dedifferentiated more malignant nature
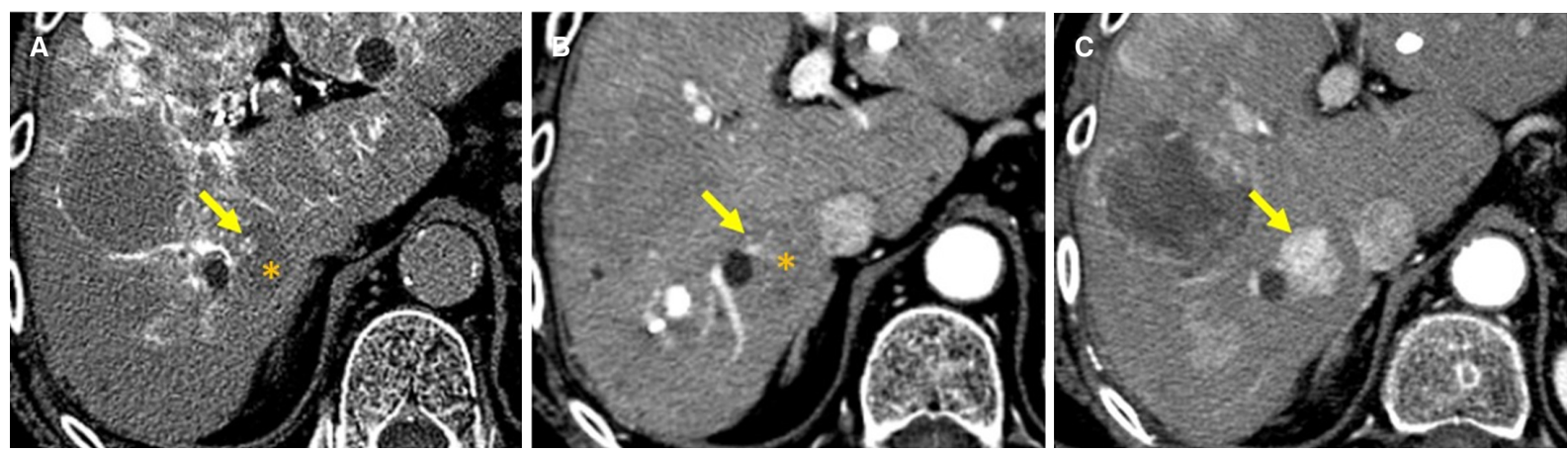

Figure 11. Development of hepatocellular carcinoma from hypovascular nodule with hypervascular foci. $A$, B: on CT during hepatic arteriography (A) and arterial phase of dynamic CT (B), small hypervascular foci (arrow) is observed within hypovascular nodule $\left({ }^{*}\right.$ ); C: eleven months after, on arterial phase of dynamic $C T$, the hepovascular nodule with hypervascular foci transformed to hypervascular hepatocellular carcinoma (arrow)

the relatively benign nature of most of the nodule. However, if nodules contain foci that show low density on CTAP but a high density on CTHA, this would represent hypervascularity and likely malignant nature of the affected portion. This type of nodule is considered visualization of the multi-step hepatocarcinogenesis process within the tumor, namely a more hypervascular and thus malignant foci develops within a hypovascular and relatively benign nodule. To find out this kind of dedifferentiated hypervascular foci within hypovascular nodule might be easy and reliable method to pick up high-risk precancerous nodule among hypovascular cirrhotic nodules.

Nodules with relatively benign features including preserved portal blood flow on CTAP and hypovascularity on CTHA vary from LGDN to early HCC that are relatively benign. In contrast, nodules that lack portal blood flow on CTAP and have an area of high density on CTHA are moderately differentiated and hypervascular HCC, which are definitely malignant nodules. The hypovascular nodule with a hypervascular foci is considered the transitional stage nodule between more benign and definitely malignant nodules [Figure 11].

Nodules with such hypervascular focis within hypovascular nodules, which is the visualization of multi-step hepatocarcinogenesis, are relatively easy to pick up on dynamic contrast enhanced CT/MR imaging [Figure 12], 

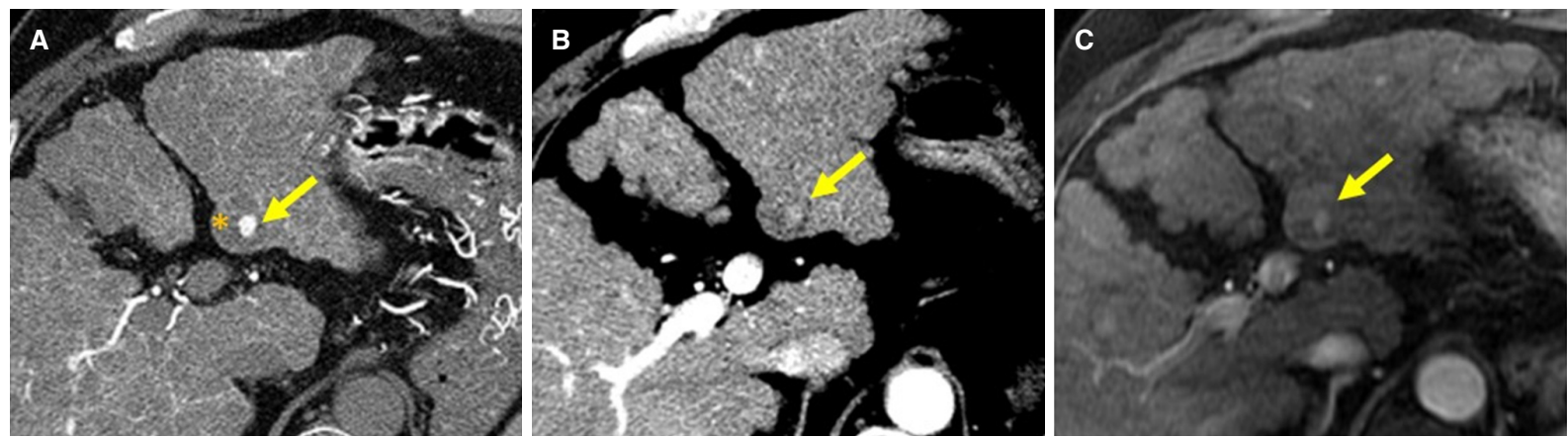

Figure 12. Visualization of hypervascular foci on dynamic contrast enhanced CT and MRI. A: on CT during hepatic arteriography, hypervascular foci (arrow) is observed within hypovascular nodule ( $\left.{ }^{\star}\right)$; B: on arterial phase of dynamic CT, small early enhancement (arrow) is observed within hypovascular nodule; C: on arterial phase of dynamic MRI with Gd-EOB-DTPA, mall early enhancement (arrow) is observed within hypovascular nodule

and can be used as a kind of potential biomarker to differentiate between benign and malignant nodules on imaging.

Until now, we have mainly reviewed the imaging findings of angiography-assisted CT. To acquire angiography-assisted CT images however, angiography, which is relatively invasive and an unusual procedure in daily clinical practice, is necessary. By comparison, intravenous contrast enhanced dynamic CT or dynamic MRI, which is usually performed in clinical practice, can also detect hypervascular foci in hypovascular nodules.

Shinmura et al. ${ }^{[12]}$ reported in 2008 that when the size of the hypervascular foci was $5 \mathrm{~mm}$ or more, dynamic CT and dynamic could detect $83 \%$ and $64 \%$ of hypervascular foci in hypovascular nodules respectively. Detection rates have improved on the latest $\mathrm{CT}$ and MR equipment but further examination is needed to clarify this issue.

\section{Approach from MRI signal intensity}

On correlation studies of MRI signal intensities with histopathological findings, DN and early HCC have iso- to high-intensity on T1-weighted images and a iso- to low-intensity on T2-weighted images ${ }^{[13]}$. while moderately differentiated HCC has low-, iso-, and high-intensity on T1-weighted images and a high-intensity on T2-weighted images [Figure 13] $]^{[14]}$. One of the reasons for various signal intensities on T1-weighted images in HCC is a higher protein content due to an increase in cell density and/or N-C ratio.

In terms of the relationship between nodular arterial and portal blood supply and MR signal intensity, nodules with normal portal blood flow but low hepatic arterial flow (estimated to be DN to early HCC) had $63 \%$ high-intensity on T1-weighted images and 72\% low-intensity on T2-weighted images; nodules which had mildly decreased portal blood flow and still low hepatic arterial flow (estimated to be early HCC to well differentiated HCC) had 39\% iso-intensity on T1-weighted images and 52\% iso-intensity on T2-weighted images; and nodules which lacked portal blood flow and had markedly high hepatic arterial flow (estimated to be moderately differentiated HCC) had $48 \%$ low-intensity on T1-weighted images and $85 \%$ high-intensity on T2-weighted images ${ }^{[15]}$.

These studies show that for estimation of the grade of malignancy in hepatocellular nodules on MRI signal intensity, T2-weighted images are useful, and the low- to iso-intensity of the nodules suggest relatively benign pathology such as RN, DN to early HCC, and high-intensity would suggest moderately differentiated HCC. 


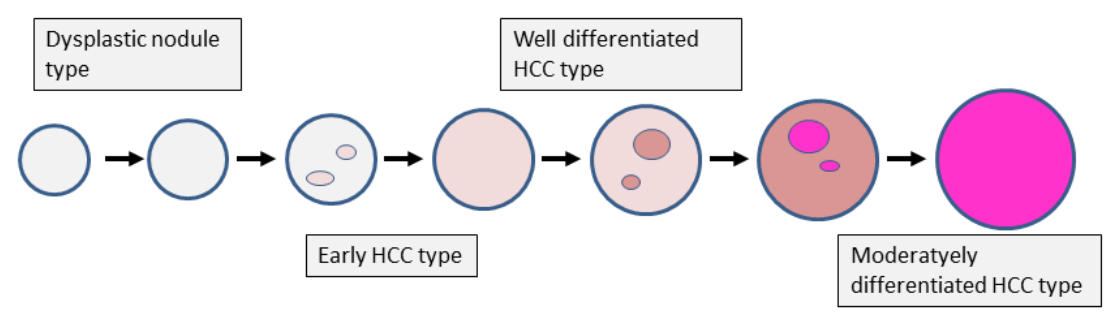

Signal intensity on T1-WI

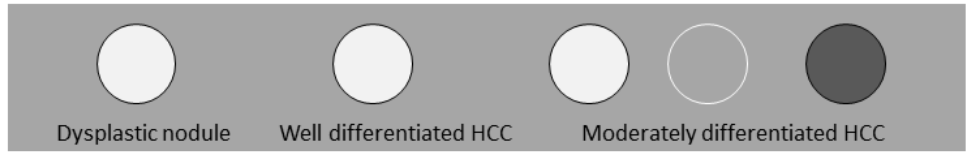

Signal intensity on T2-WI

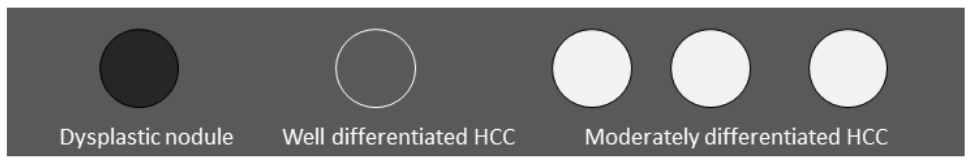

Figure 13. MR signal intensity change during multistep hepatocarcinogenesis. HCC: hepatocellular carcinoma

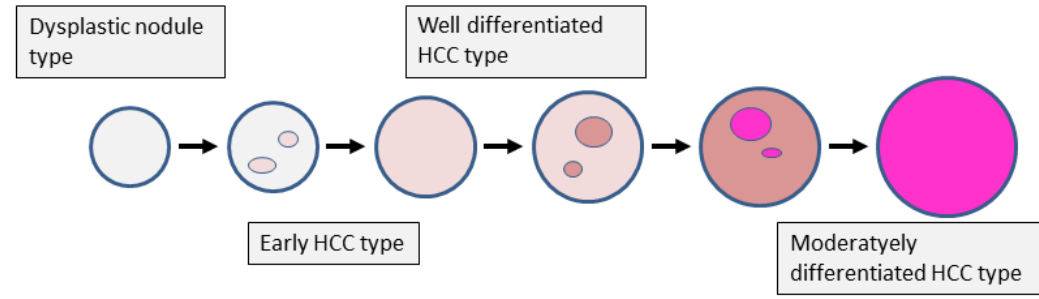

Kupffer cell count ratio

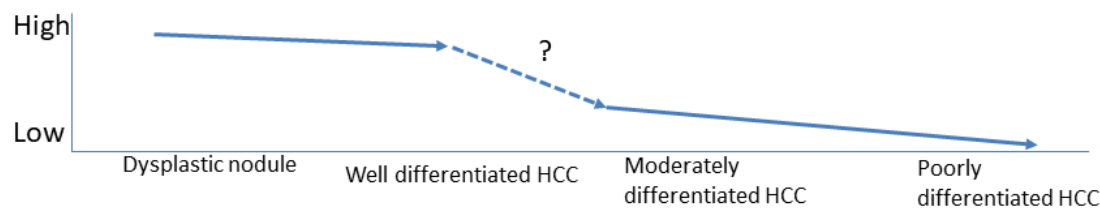

Signal intensity on SPIO enhanced T2-WI

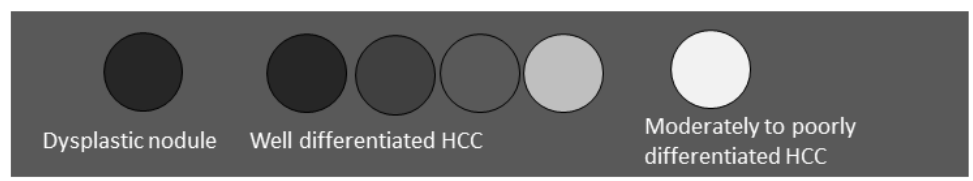

Figure 14. SPIO enhanced T2 weighted MR signal intensity change during multistep hepatocarcinogenesis. HCC: hepatocellular carcinoma; SPIO: superparamagnetic iron oxide

\section{Approach from functional evaluation of Kupffer cells}

Sinusoids are where blood flows through the liver cords. It consists of sinusoidal endothelial cells, Kupffer cells, stellate cells, and pit cells ${ }^{[16]}$. Kupffer cells are intravascular resident macrophages in liver sinusoids. They are capable of phagocytosis and eliminate foreign bodies, bacteria, and broken hepatocytes.

When SPIO is administered intravenously in the normal liver, Kupffer cells in sinusoids take it up. SPIO shortens the transverse relaxation time of protons of tissues, which results in low signal intensities on T2- 
weighted MR images. In cases where the normal structure of sinusoids is lost (metastatic liver cancer etc.), signal intensity changes do not occur because there are no Kupffer cells. Similarly, the function of Kupffer cells are decreased despite their presence due to irradiation etc., the degree of SPIO uptake is lowered, and such areas show relatively high signal intensities on T2-weighted images compared to the surrounding normal liver where normal Kupffer cells are present and take up SPIO ${ }^{[17]}$.

On histopathological analysis, Imai et al. ${ }^{[18]}$ found that in multi-step hepatocarcinogenesis, as the nodular degree of differentiation is lowered, the number of Kupffer cells within the nodule is decreased, and there was a significant difference between the number of Kupffer cells in DN, well differentiated HCC, moderately differentiated HCC, and poorly differentiated HCC. In most DN and $46 \%$ of well differentiated HCC, the number of Kupffer cells was increased compared with the surrounding liver; the number of Kupffer cells decreased to $30 \%$ compared with the surrounding liver in moderately differentiated HCC; and the number of Kupffer cells decreased to $13 \%$ on average in poorly differentiated HCC [Figure 14].

On SPIO-enhanced MRI, DN and well-differentiated HCC present with iso- to slightly low-intensity on T2 weighted MR images compared to the surrounding liver. In well-differentiated HCC especially, 46\% of the nodules presented with a low-intensity compared to the surrounding liver. Moderately and poorly differentiated HCC though presented with a high-intensity compared with the surrounding liver. There was a significant difference in signal intensity between DN with well-differentiated HCC, and moderately and poorly differentiated HCC [Figure 14] ${ }^{[18]}$. These results show that SPIO-enhanced MRI is a good tool to estimate the degree of differentiation in HCC, but it has a limit in differentiating DN from well-differentiated HCC.

\section{Approach from the function of hepatocyte membrane transporter}

Approximately 50\% of Gd-EOB-DTPA (EOB), as well as other extracellular contrast agents, is excreted from the kidneys and the remaining $50 \%$ is taken up by hepatocytes and then excreted into the biliary tract, hence, it is called hepatocyte-specific contrast agent. The advantage of this MRI contrast agent is it can evaluate both blood flow information and hepatocyte function simultaneously. However, in EOB-enhanced MRI, there are some drawbacks of low accumulation in hepatocytes depending on liver function and the low degree of arterial enhancement, and there are also limitations in viewing true washout or enhancement of capsules, which are major features characterizing overt HCC.

After rapid intravenous injection of EOB, blood flow evaluation by dynamic study is performed similarly to other extracellular contrast agents. In the hepatobiliary phase after 15-20 min of injection, the liver parenchyma presents high signal intensity on T1-weighted images because of EOB uptake by normal hepatocytes. Areas that do not have normal hepatocytes (metastatic liver cancer etc.) or areas where hepatocytes have poor EOB uptake ability (irradiated area etc.) present with low intensities compared to the surrounding normal liver.

EOB is taken up into the intracellular space of hepatocytes by organic anion transporting polypeptide (OATP) 1B3, which is expressed on the sinusoidal side of hepatocyte membranes and excreted in the biliary tract through multidrug resistance associated protein 2 (MRP2), which is expressed on the bile canalicular side of hepatocyte membrane ${ }^{[19]}$. Under the conditions of bile duct obstruction, MRP3, which is expressed on the sinusoidal side of hepatocyte membranes, increase and EOB is excreted into the sinusoids ${ }^{[19]}$. Kitao et al. ${ }^{[20]}$ compared the degree of OATP1B3 expression in hepatocellular nodules undergoing multistep hepatocarcinogenesis with the surrounding normal liver, and found that it was preserved in LGDN but decreased in approximately $30 \%$ of HGDN and approximately $70 \%$ of early HCC [Figure 15].

In most moderately differentiated HCC, there was decreased expression of OATP1B3. As well, in the hepatobiliary phase of EOB-enhanced MRI, the signal enhancement ratio was significantly lowered in well to 

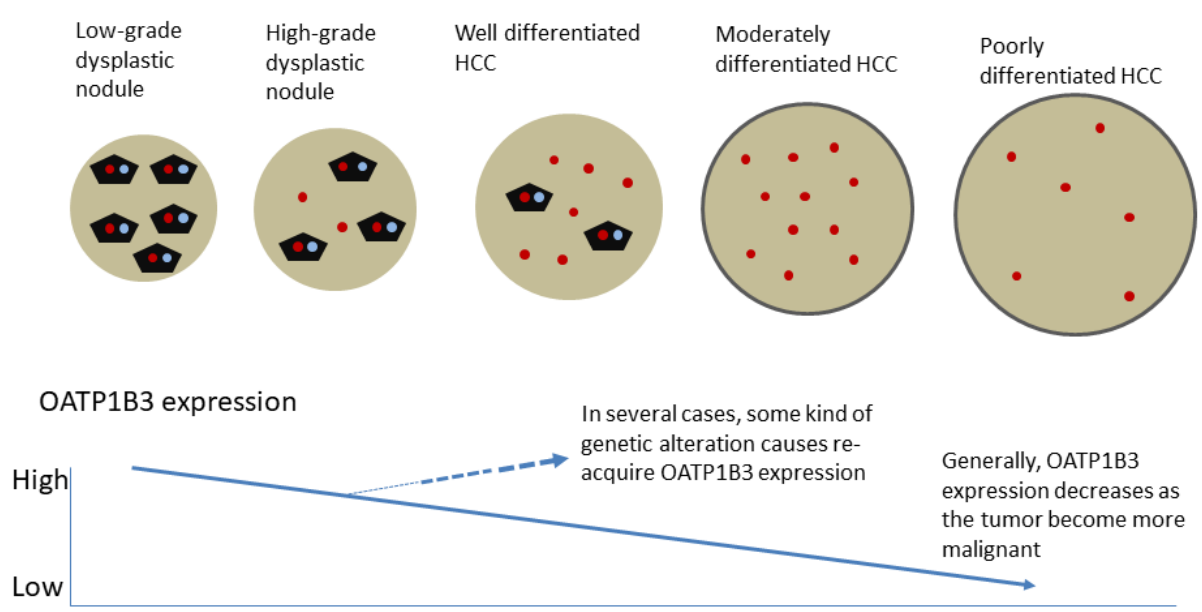

\section{EOB uptake shown on MRI}

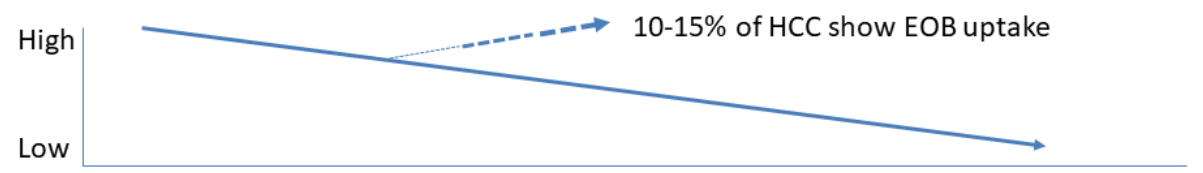

Figure 15. Relationship between OATP1B3 expression, EOB uptake and multistep hepatocarcinogenesis. HCC: hepatocellular carcinoma; EOB: Gd-EOB-DTPA

moderately differentiated HCC compared to the surrounding liver. As the grade of malignancy increases from well-differentiated to moderately- and poorly-differentiated HCC, the signal enhancement ratio decreases. However, in 10\%-15\% of moderately differentiated HCC, there was increased expression of OATP1B3 and these HCC present a high signal in the hepatobiliary phase of EOB-enhanced MRI [Figure 15].

Sano et al. ${ }^{[21]}$ compared the ability of various imaging modalities, including dynamic CT, angiographyassisted CT, and EOB-enhanced MRI, in differentiating between histologically confirmed DN and early HCC. They showed that in the hepatobiliary phase of EOB-enhanced MRI, there were no DN nodules which presented with low-intensity whereas $97 \%$ of early HCC presented with low-intensity, and concluded this was best suited for the differential diagnosis of DN and early HCC. We surmise then that before starting histopathological and/or morphological malignant transformation observed as microscopic stromal invasion, functional alterations of cellular components such as membranous transporter functional states, which is regulated by genome level alterations, are already advanced within the nodule and can be detected by EOB. Further study is needed though to clarify this issue.

We also studied the relationship between the signal intensity of nodules during the hepatobiliary phase of EOB-enhanced MRI with the prognosis of these nodules that were smaller than $20 \mathrm{~mm}$ (average size and SD; $11.0 \pm 2.8 \mathrm{~mm}$ ), both in hypovascular ones (relatively more benign nature nodule) in multi-step hepatocarcinogenesis and in hypovascular ones with hypervascular foci (hypervascular foci-containing nodules) that are more representative of malignancy. Hypovascular nodules with a low-intensity in the hepatobiliary phase of EOB-enhanced MRI,progressed to hypervascular HCC in 17\%, 28\% and 41\% after 1, 2 and 3 years respectively, while those with iso-intensity only progressed to hypervascular HCC in 7\% after 1 year and those with high-intensity did not progress at all ${ }^{[22]}$.

On the other hand, in hypervascular foci-containing nodules, whether the signal intensity was low or iso during the hepatobiliary phase of EOB-enhanced MRI, 50\% progressed to hypervascular HCC after 1 year ${ }^{[23]}$. This suggests that hypervascular foci in nodules can exacerbate multi-step hepatocarcinogenesis regardless of the decrease in $\mathrm{EOB}$ uptake. 


\section{CONCLUSION}

In this article, we have reviewed how malignant nodules can be differentiated from benign ones in multistep hepatocarcinogenesis using various imaging techniques. It is difficult to draw a clear line between them however, because different imaging modalities reflect different pathophysiology and biopsies could have sampling errors. However, this review would have helped in understanding of the current status in diagnosing hepatocellular nodules, whether benign or malignant, in various imaging modalities.

\section{DECLARATIONS}

\section{Authors' contributions}

Design of the review: Matsui O, Kobayashi S

Literature review and Manuscript writing: Kobayashi S, Kozaka K, Minami T

Manuscript revision: Kobayashi S, Gabata T

\section{Availability of data and materials}

Not applicable.

\section{Financial support and sponsorship}

None.

\section{Conflicts of interest}

All authors declared that there are no conflicts of interest.

\section{Ethical approval and consent to participate}

Not applicable.

\section{Consent for publication}

Not applicable.

\section{Copyright}

(c) The Author(s) 2020.

\section{REFERENCES}

1. Torre LA, Bray F, Siegel RL, Ferlay J, Lortet-Tieulent J, et al. Global cancer statistics. 2012. CA Cancer J Clin 2015;65:87-108.

2. Ichikawa S, Ichikawa T, Motosugi U, Sano K, Morisaka H, et al. Was hypervascular hepatocellular carcinoma visible on previous gadoxetic acid-enhanced magnetic resonance images? Liver Cancer 2015;4:154-62.

3. Tamada T, Korenaga M, Yamamoto A, Higaki A, Kanki A, et al. Assessment of clinical and magnetic resonance imaging features of de novo hypervascular hepatocellular carcinoma using gadoxetic acid-enhanced magnetic resonance imaging. Hepatol Res 2017;47:E15260 .

4. Sakamoto M, Effendi K, Masugi Y. Molecular diagnosis of multistage hepatocarcinogenesis. Jpn J Clin Oncol 2010;40:891-6.

5. International Consensus Group for Hepatocellular NeoplasiaThe International Consensus Group for Hepatocellular Neoplasia. Pathologic diagnosis of early hepatocellular carcinoma: a report of the international consensus group for hepatocellular neoplasia. Hepatology 2009;49;658-64.

6. Torbenson MS, Ng IOL, Park YN, Sakamoto M. Hepatocellular carcinoma. In: edited by the WHO Classification of Tumours Editorial Board. WHO Classification of Tumours. 5th edition. Digestive System Tumours. Lyon, France: International Agency for Research on Cancer; 2019. pp. 229-39.

7. Nakano M, Saito A, Yamamoto M, Doi M, Takasaki K. Stromal and blood vessel wall invasion in well-differentiated hepatocellular carcinoma. Liver 1997; 17:41-6.

8. Ueda K, Terada T, Nakanuma Y, Matsui O. Hum Pathol 1992;23:619-26.

9. Hayashi M, Matsui O, Ueda K, Kawamori Y, Kadoya M, et al. Correlation between the blood supply and grade of malignancy of hepatocellular nodules associated with liver cirrhosis: evaluation by $\mathrm{CT}$ during intraarterial injection of contrast medium. Am $\mathrm{J}$ Roentogenol 1999;172:969-76.

10. Kitao A, Zen Y, Matsui O, Gabata T, Nakanuma Y. Hepatocarcinogenesis: multistep changes of drainage vessels at CT during arterial 
portography and hepatic arteriography--radiologic-pathologic correlation. Radiology 2009;252:605-14.

11. Ueda K, Matsui O, Kawamori Y, Nakanuma Y, Kadoya M, et al. Hypervascular hepatocellular carcinoma: evaluation of hemodynamics with dynamic CT during hepatic arteriography. Radiology 1998;206:161-6.

12. Shinmura R, Matsui O, Kadoya M, Kobayashi S, Terayama N, et al. Detection of hypervascular malignant foci in borderline lesions of hepatocellular carcinoma: comparison of dynamic multi-detector row CT, dynamic MR imaging and superparamagnetic iron oxideenhanced MR imaging. Eur Radiol 2008;18:1918-24.

13. Matsui O, Kadoya M, Kameyama T, Yoshikawa J, Arai K, et al. Adenomatous hyperplastic nodules in the cirrhotic liver: differentiation from hepatocellular carcinoma with MR imaging. Radiology 1989;173:123-6.

14. Kadoya M, Matsui O, Takashima T, Nonomura A. Hepatocellular carcinoma: correlation of MR imaging and histopathologic findings. Radiology 1992;183:819-25.

15. Shinmura R, Matsui O, Kobayashi S, Terayama N, Sanada J, et al. Cirrhotic nodules: association between MR imaging signal intensity and intranodular blood supply. Radiology 2005;237:512-9.

16. Abu Rmilah A, Zhou W, Nelson E, Lin L, Amiot B, et al. Understanding the marvels behind liver regeneration. Wiley Interdiscip Rev Dev Biol 2019;8:e340.

17. Kawamori Y, Matsui O, Kadoya M, Yoshikawa J, Demachi H, et al. Differentiation of hepatocellular carcinomas from hyperplastic nodules induced in rat liver with ferrite-enhanced MR imaging. Radiology 1992;183:65-72.

18. Imai Y, Murakami T, Yoshida S, Nishikawa M, Ohsawa M, et al. Superparamagnetic iron oxide-enhanced magnetic resonance images of hepatocellular carcinoma: correlation with histological grading. Hepatology 2000;32:205-12.

19. Kitao A, Zen Y, Matsui O, Gabata T, Kobayashi S, et al. Hepatocellular carcinoma: signal intensity at gadoxetic acid-enhanced MR Imaging--correlation with molecular transporters and histopathologic features. Radiology 2010;256:817-26.

20. Kitao A, Matsui O, Yoneda N, Kozaka K, Shinmura R, et al. The uptake transporter OATP8 expression decreases during multistep hepatocarcinogenesis: correlation with gadoxetic acid enhanced MR imaging. Eur Radiol 2011;21:2056-66.

21. Sano K, Ichikawa T, Motosugi U, Sou H, Muhi AM, et al. Imaging study of early hepatocellular carcinoma: usefulness of gadoxetic acidenhanced MR imaging. Radiology 2011;261:834-44.

22. Kobayashi S, Matsui O, Gabata T, Koda W, Minami T, et al. Relationship between signal intensity on hepatobiliary phase of gadolinium ethoxybenzyl diethylenetriaminepentaacetic acid (Gd-EOB-DTPA)-enhanced MR imaging and prognosis of borderline lesions of hepatocellular carcinoma. Eur J Radiol 2012;81:3002-9.

23. Kobayashi S, Matsui O, Gabata T, Koda W, Minami T, et al. Gadolinium ethoxybenzyl diethylenetriamine pentaacetic Acid-enhanced magnetic resonance imaging findings of borderline lesions at high risk for progression to hypervascular classic hepatocellular carcinoma. J Comput Assist Tomogr 2011;35:181-6. 